\title{
In-Game Peer Performance Assessment Role That Fosters Metacognitive Agility and Reflection
}

\author{
Elaine M. Raybourn \\ * Sandia National Laboratories, P.O. Box 5800 MS 1188 \\ Albuquerque, New Mexico USA, 87185 \\ emraybo@sandia.gov
}

\begin{abstract}
In this paper we describe the development of a method and system for training metacognitive agility (self-awareness and self-regulated learning) in serious games applications. We introduce a unique design that features a novel role for real-time, in-game peer performance assessment and feedback to encourage user reflection and self-explanation. This approach has been implemented in two serious games currently in use today whose focus is intercultural competence and intercultural sensitivity education.
\end{abstract}

Keywords: serious game, metacognitive agility, reflection, in-game performance assessment, peer learning, intercultural competence, sensitivity.

\section{Reflective Evaluator Role in Multiplayer Serious Games}

A serious game is defined in this paper as the use of interactive digital technologies for training and education in private, public, government, and military sectors. In multiplayer serious games play is usually followed by a debriefing period in which key learning points and participant performance are discussed in a large group after an exciting game session. It is typical in these cases, therefore, for the reflection and peer learning to occur outside of the game experience, while the game experience focuses on individual task performance [1]. It is also typical to hear these users and instructors say that the real learning occurs outside of the game, not as part of the game. However, this trend need not persist. By applying principles from social learning theory and entertainment game design we can embed in-game opportunities for user real-time reflection and peer learning during game play.

Honing one's metacognitive agility and reflection is integral to competence education [1]. Metacognitive agility is the ability to actively control the learning process [2] such as possessing the ability to reflect and analyze the way oneself or others think, discern when different cognitive strategies are needed, and employ those strategies to enhance one's learning and performance [1]. Operating competently requires the

\footnotetext{
Sandia is a multiprogram laboratory operated by Sandia Corporation, a Lockheed Martin Company, for the United States Department of Energy under Contract DE-AC0494AL85000.
} 
ability to be aware of oneself and others, reflect on salient experiences, evaluate or assess situations, and act purposefully on those evaluations.

The reflective evaluator role is based on the Real-time In-Game Assessment, Evaluation and Feedback system [1,3] which consists of a game interface and role for making in-game evaluations of player's actions, decisions, communications, etc. as they occur in real-time and as they correspond to competencies and learning objectives. In each multiplayer session, Trainees, instructors, evaluators, or subject matter experts play roles for characters or peer observer/evaluators.

Our design goal with the introduction of this new role for reflective learning (observation and evaluation) was to encourage growth toward intercultural sensitivity [4, 3] and cultural relativism [5], or the ability to reflect and evaluate phenomena relative to cultural context. Therefore our intent was to expand trainees' solution set for ill-defined problems through their game session and subsequent participation in a follow-up discussion. More detailed information on the role is available from [1, 3].

Peers in the reflective role help focus and teach others participating in the debriefing to connect theories and/or concepts to actions demonstrating stages of intercultural competence and sensitivity [3,4]. Trainees in the reflective role are therefore held accountable for explaining criteria of intercultural competence to others after observing modeled behaviors. This often accelerates learning as they internalized concepts and new vocabulary more quickly than others.

By training in roles that allow users to act (character roles) and reflect (Reflective Evaluator role) the trainees perform different cognitive tasks. More concrete, active experimentation (e.g. negotiating from a different point of view) takes place with character role participation, while abstract conceptualization and reflection is fostered by the Reflective Evaluator role (e.g. pause, look at the problem in light of the cultural context, critically consider best practices, and communicate solutions to others). Thus intercultural competence education is grounded in experiential learning theory-namely concrete experience, active experimentation, reflective observation, and abstract conceptualization [6]. This approach is entertaining and educational. It is used with multicultural groups to engender out-of game discussions of cultural differences and diverse approaches to problem-solving in two serious games currently in use today. We are extending this work by conducting user studies that investigate how to further improve experiential learning and by adapting the game environment in real-time as a result of performance, reflection, and in-game peer evaluation.

\section{References}

1. Raybourn, E.M.: Training approaches for honing junior leader adaptive thinking, cultural awareness and metacognitive agility. In: Proc. I/ITSEC 2007, Interservice/ Industry Training, Simulation and Education Conference, Orlando, Florida, USA (2007)

2. Flavell, J.H.: Metacognition and cognitive monitoring: A new area of cognitivedevelopmental inquiry. American Psychologist 34, 906-911 (1979)

3. Raybourn, E.M.: Applying simulation experience design methods to creating serious gamebased adaptive training systems. Interacting with Computers 19, 207-214 (2007) 
4. Bennett, M.J.: A developmental approach to training for intercultural sensitivity. International Journal of Intercultural Relations 10, 179-196 (1986)

5. Rogers, E.M., Steinfatt, T.M.: Intercultural Communication. Waveland Press, Inc., Prospect Heights (1999)

6. Kolb, D.A.: Experiential learning: Experience as the Source of Learning and Development. Prentice-Hall, NJ (1984) 